\title{
ZL-130TCII FRACTURE MARKERS SOLUTION FOR FULL-SCALE FATIGUE TEST
}

\author{
Sylwester Kłysz \\ Janusz Lisiecki \\ Dariusz Zasada \\ Gabriel Gmurczyk \\ Andrzej Leski \\ Institute of Aeronautics and Applied Mechanic, \\ Warsaw, Poland
}

\begin{abstract}
In the context of PZL-130TC-II full-scale fatigue test, several strategies of fatigue loadings that create fracture surface markings were considered. One block of spectrum is made of 200 flights. By reordering those flights, a block which should create a fracture marker, was developed. It was very important that reordering the load spectrum or adding overloads or underloads did not change spectrum severity. Pilot tests of aluminium alloys specimens were carried out to finalise appropriate marker intervals before commencing full-scale tests. The experiment was conducted with the MTS machine with 810.23 system. The results and conclusions are presented within this paper.
\end{abstract}

Keywords: full scale fatigue test, fracture surface marking, Quantitative Fractography

\section{INTRODUCTION}

One of the purposes of the full-scale fatigue test (FSFT) is to provide information on the location and character of fatigue crack propagation. FSFT usually produces a few large cracks and many smaller ones. After applying loads, during Teardown Inspection (TI) there are found cracks which are not visible. There is no information about their history (crack growth rate, time of crack initiation and number of cycles). In such cases, post-test Quantitative Fractography (QF) of crack growth is needed. This method requires fracture surface markers, which are formed naturally or by intentionally developed load spectrum. The occurrence of markers depends on several factors: material, spectrum (loads, $\mathrm{R}$ ratio, intervals, peaks), environmental conditions, crack size and crack growth rate. The marker load strategies fall into five main categories:

- reordering the load spectrum

- overload additions

- underload additions

- constant amplitude (CA) groups

- combination of the above 


\section{REORDERING THE GROUP OF LOADS}

One block of spectrum is made of 198 flights. It is equivalent to 200 flight hours. By reordering those flights, a block which should create a fracture marker was developed. It was very important not to change spectrum severity by reordering or adding load peaks. A reordered load spectrum is shown in Fig. 1.

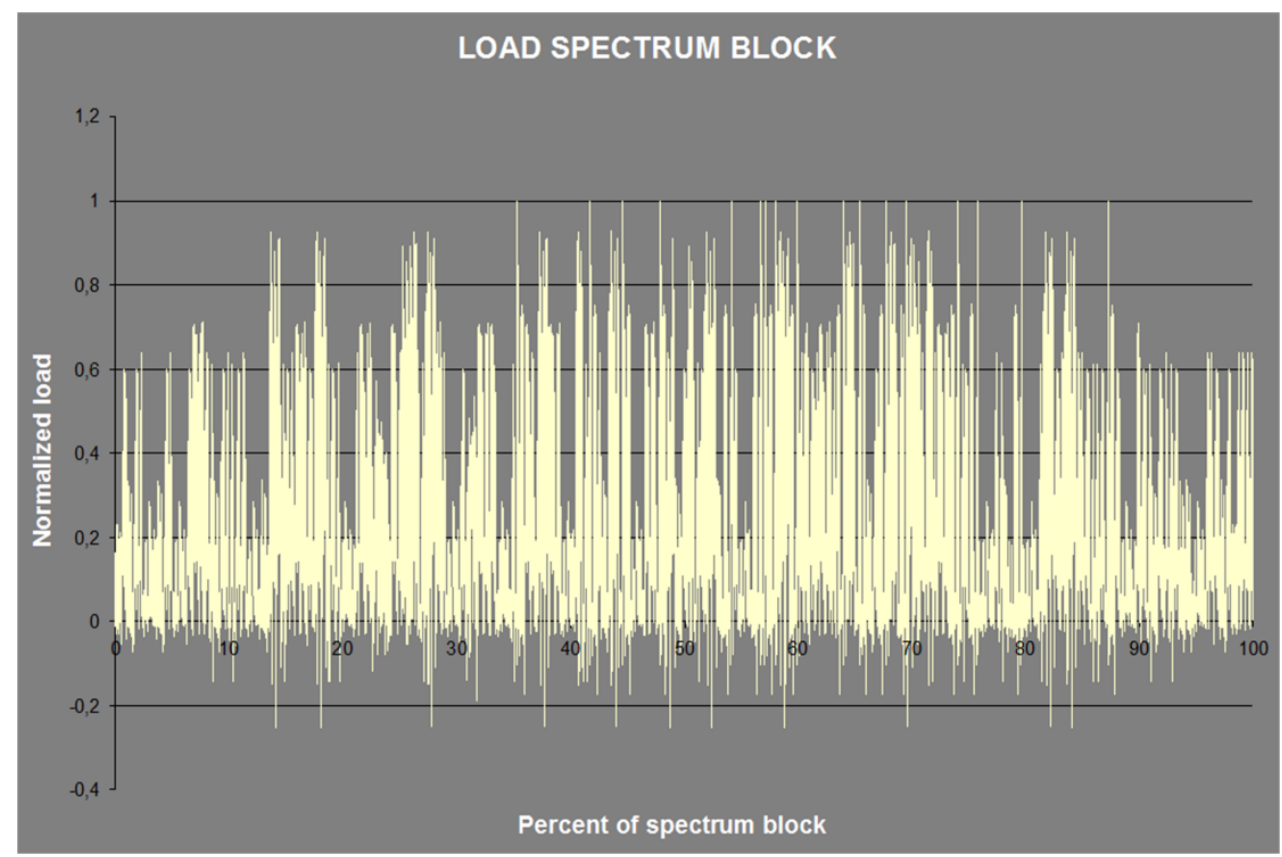

Fig. 1. Reordered load spectrum (one block)

\section{PILOT TESTS}

Studies with the use of reordered load spectrum were performed on WOL-type aluminum alloy specimens - shown in Fig. 2. After precracking in a standard way, in successive stages, the tests were conducted at the above spectrum and controlling the size of the load spectrum amplitudes by multiplying the normalized load (Fig.1) by an appropriate scaling factor load $k_{\text {load }}$. This allowed determining the level of the maximum load $\mathrm{P}_{\mathrm{m}}$ in different blocks/phases of the loads.
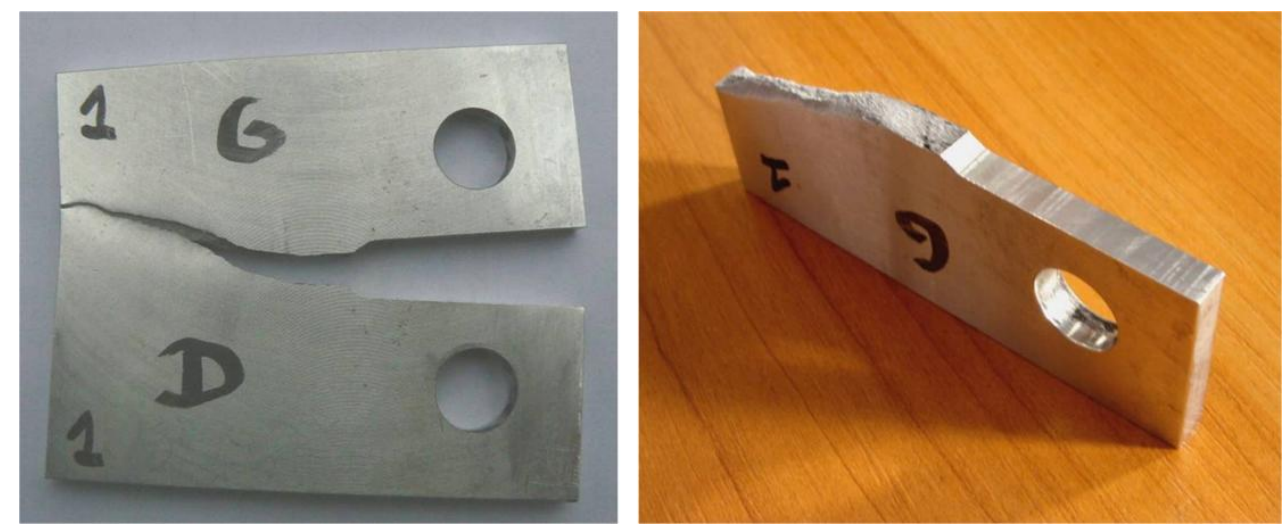

Fig. 2. WOL (Wedge Opening Load) specimen after test 


\section{Specimen No. 1.}

The first sample was placed under the tests in two phases, at different values of scaling factors $k_{\text {load }}-1.25$ and 1.50 , i.e. at loads increased by $25 \%$ and $50 \%$ compared to the force in the last cycle of the precracking stage.

Preckracking parameters:

- Number of cycles: 67532; Force in the last cycle: $P_{\text {final }}=3946 \mathrm{~N}$

- Stable crack growth under $D K$-decreasing conditions, even distribution of marker bands.

The image of the surface fatigue crack and its description are shown in Fig. 3. Fig. 4 presents the scrap microstructure at magnifications of 2000x and 10000x.
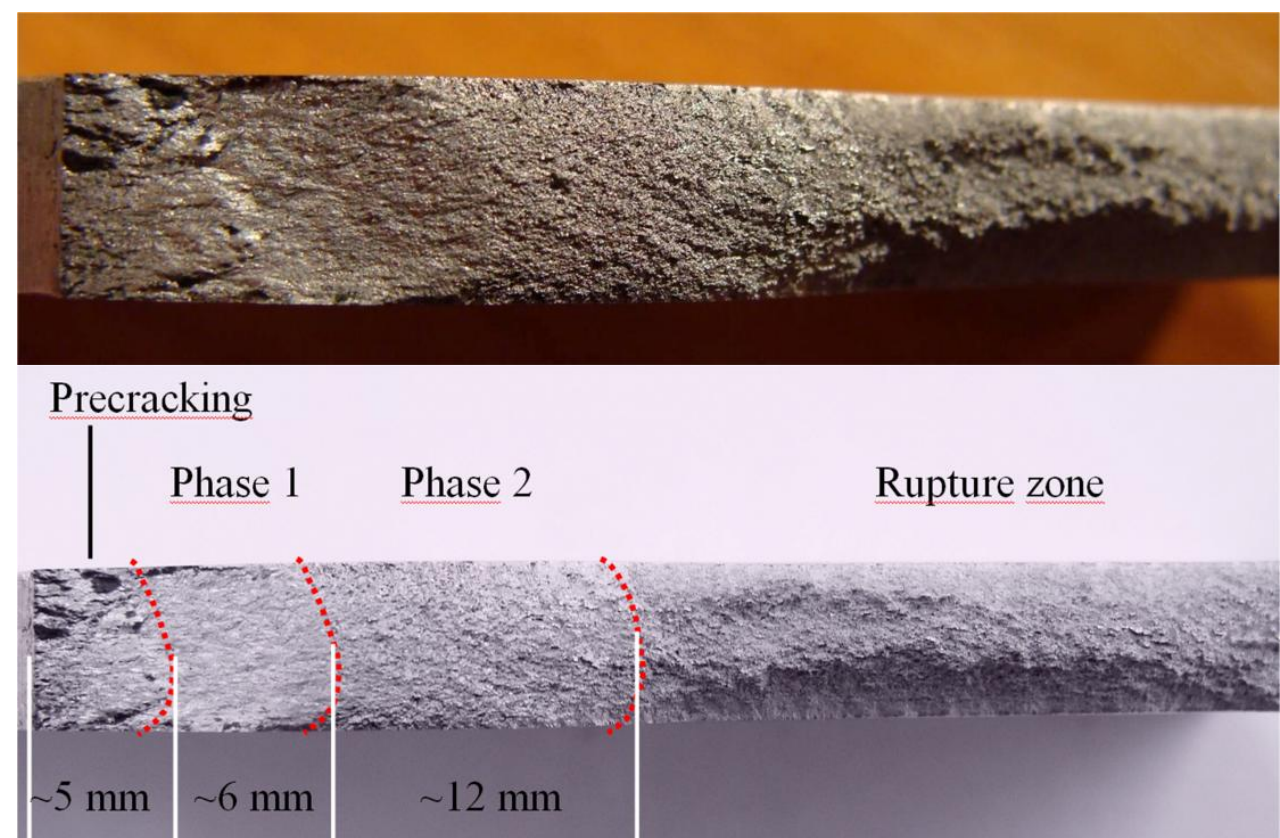

Fig. 3 Macro-fracture surface

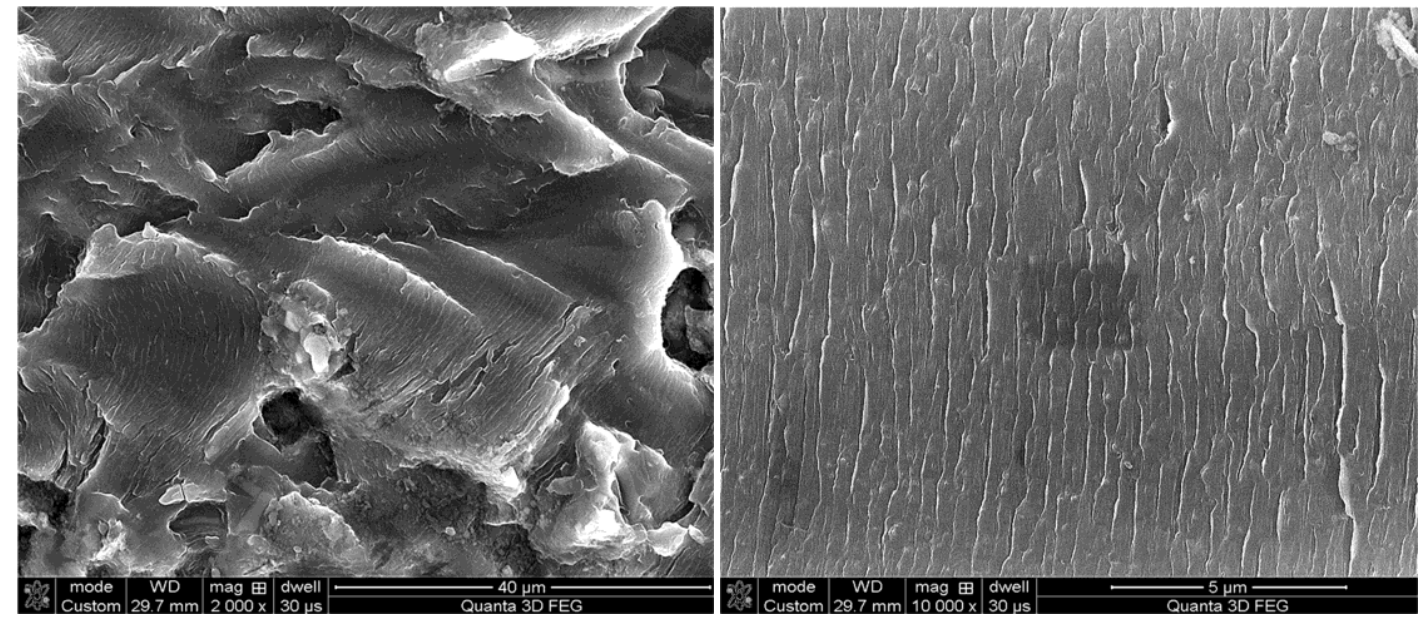

Fig. 4. SEM image micro-fracture surface

Phase 1

- Number of cycles: 1182743

- Number of blocks: 98

- Maximum force: $P_{m, I}=P_{\text {final }} * 1,25$

- Duration of block: $92,45 \mathrm{~min}$ 
Two full spectrum blocks are visible. The arrows point to characteristic areas with markings caused by the same load peaks.
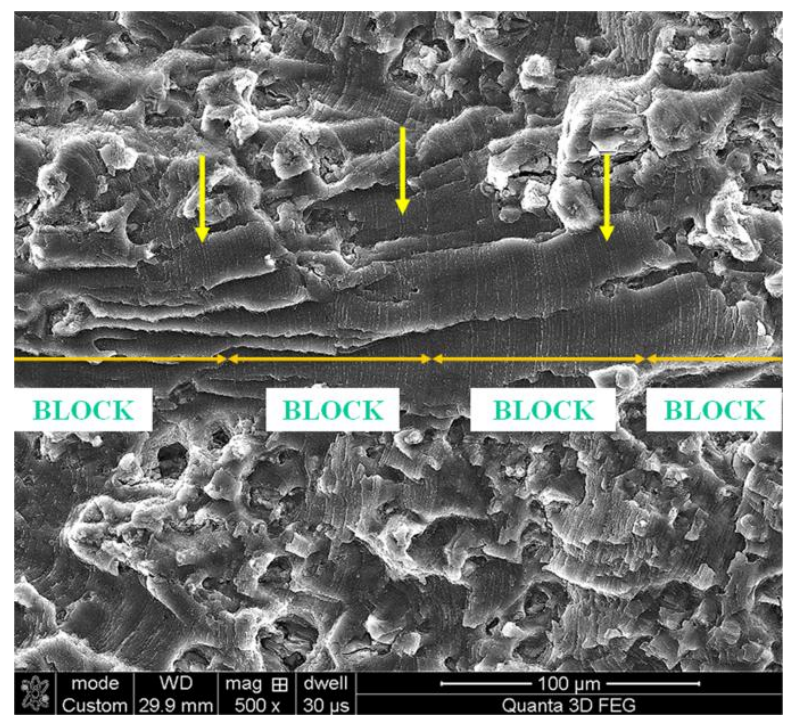

Fig. 5. SEM image of fatigue fracture surface - phase 1

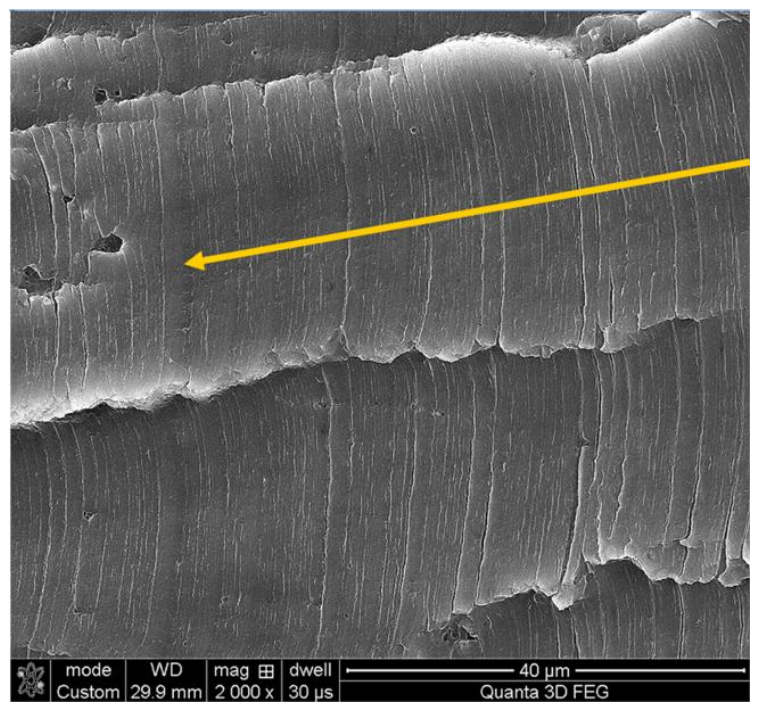

Fig. 6. SEM image of repeat markings on a fracture surface, as a result of applying spectrum - phase 1

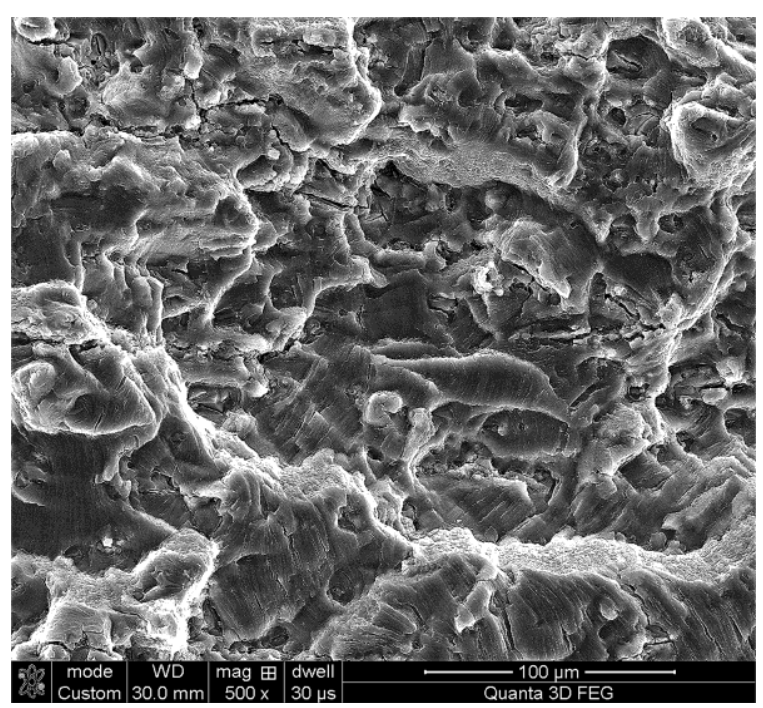

Fig. 7. SEM image of fatigue fracture surface-phase 2 
Phase 2

- Number of cycles: 63743

- Number of blocks: 4

- Maximum force: $P_{m, I I}=P_{\text {final }} * 1.5$

- Duration of block: $92,45 \mathrm{~min}$

Higher crack growth rate than in Phase 1.

More ductile tearing causes problems with analysis of fracture markings.

Fig. 8. compares Phase 1 and Phase 2 focusing on the facets. The images correspond to the maximum forces: 4932 and $5919 \mathrm{~N}$ respectively.

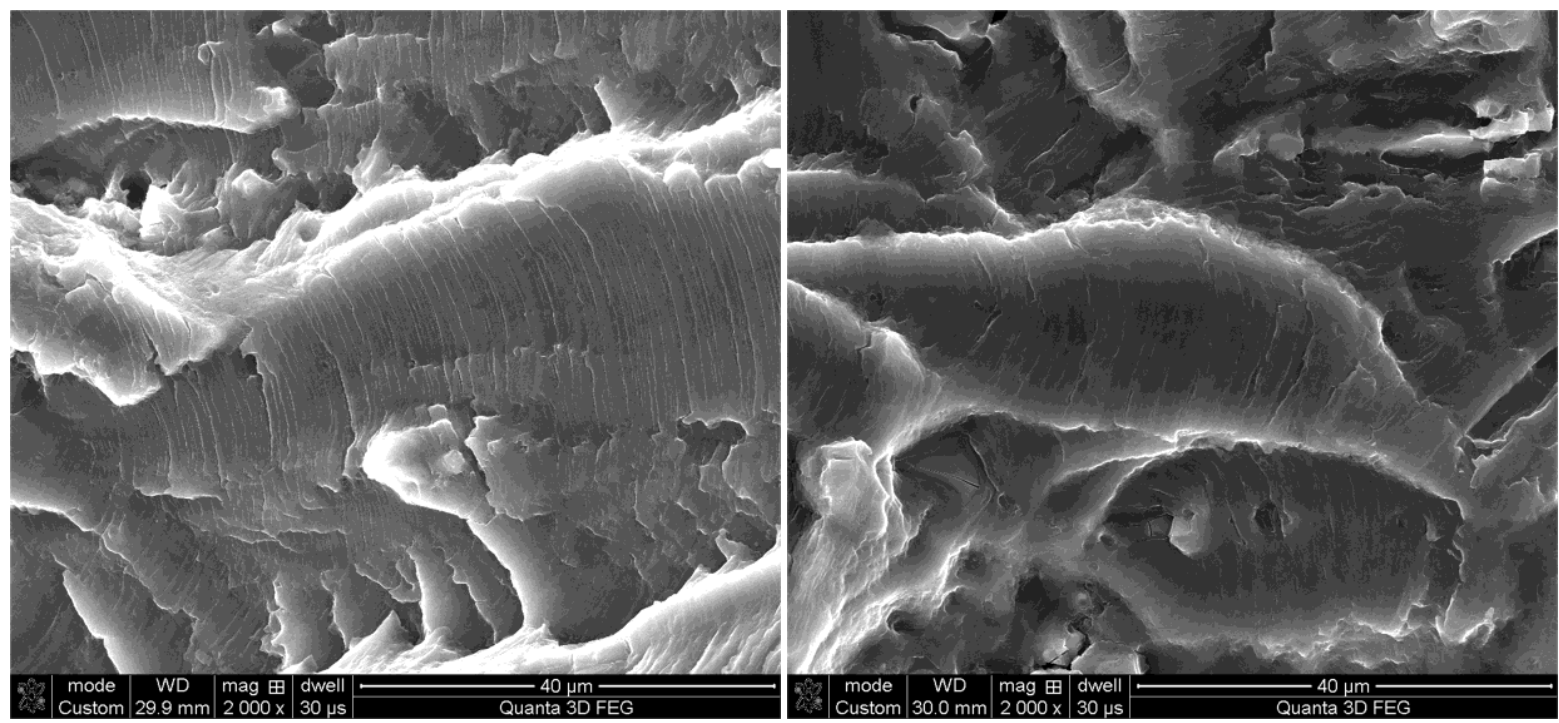

Fig. 8. SEM images of fatigue fracture surface - phase 1 and 2

\section{Specimen no. 2}

The research was conducted under conditions of reordered spectrum and overload additions according to the scheme shown in Fig.9. Another 5 cycles of constant amplitude $k_{\text {load }}=0.8$ and 25 cycles of constant amplitude $k_{\text {load }}=0.6$, for every 5 blocks of reordered spectrum were added.

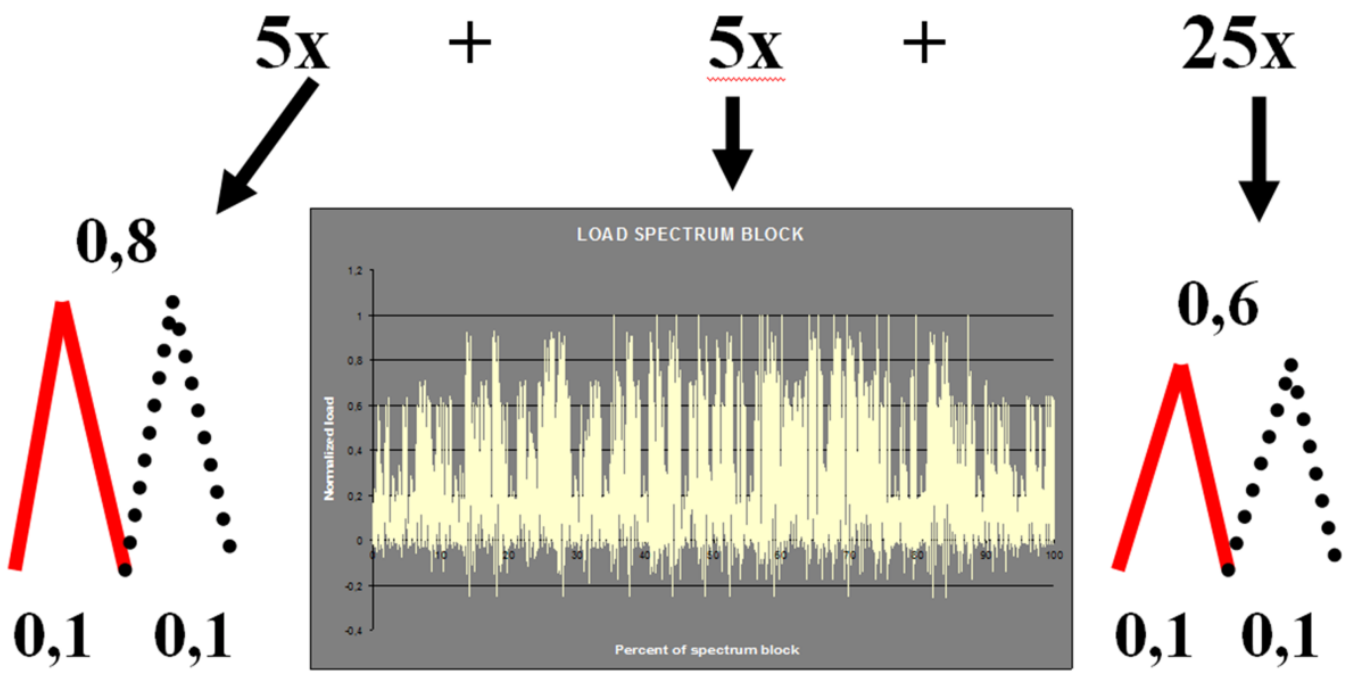

Fig. 9. Spectrum for specimen No. 2 schema 
The microstructure images of specimen no.2 are shown in Fig.10. One can see similar fatigue bands to those of specimen no.1, however the mapping of the additional cycle is not clearly identifiable in the photos. It is assumed that loads for specimen No. 2 were too low to find fracture markers.

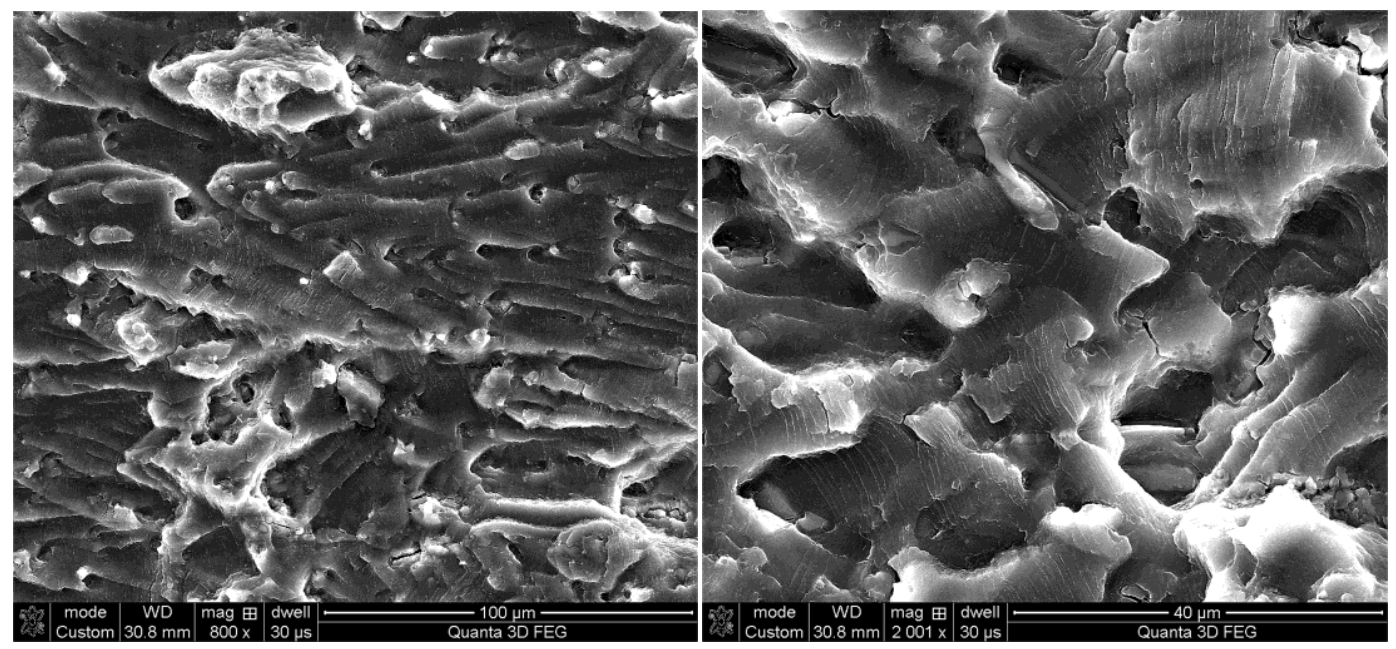

Fig. 10. SEM images of fatigue fracture surface of specimen no. 2

Therefore, it seems necessary to conduct research in other spectral load variants, modified for imaging the fracture fatigue load history in the form of controlled bands. In particular, they should include the cases with the added markers for negative cycle asymmetry coefficient $\mathrm{R}$, with markers to overload the tensile and compressive strength, with markers resulting from the reconstruction of block loads corresponding to 200 flight hours. The results of tests conducted at the AFIT in this regard will be presented in the following publications.

\section{RESULTS}

The experiments aim at finding the best solution for fracture markers for FSFT of PZL 130 Orlik TCII, which should give us information about crack history.

The difficulty in achieving high efficiency with this research method is such that laboratory simulations, accelerated in relation to the natural fatigue destruction, are necessarily conducted at higher levels of service charges, which results in increased plastic cracking in the test samples and a significant qualitative change in the microstructure image.

\section{REFERENCES}

[1] Barter, A.A., Molent, L. \& Wanhill, R.J.H (2009). Marker loads for quantitative fractography of fatigue cracks in aerospace alloys. In $25^{\text {th }}$ ICAF: Bridging the Gap between Theory and Operational Practice. 27 - 29 May 2009. Rotterdam, The Netherlands. 